\title{
OSCILLATION CRITERIA FOR SECOND-ORDER DIFFERENTIAL SYSTEMS
}

\author{
SHAIR AHMAD AND C. C. TRAVIS
}

\begin{abstract}
Sufficient conditions for the oscillation of solutions to the differential system $X^{\prime \prime}(t)+A(t) X(t)=0$ are established which are valid when the matrix $A$ is not symmetric. An example is given to demonstrate that a condition known to be sufficient for the oscillation of solutions when $A$ is symmetric is not valid in the nonsymmetric case.
\end{abstract}

There is a rather extensive literature on the Sturmian propererties of second-order differential systems of the form

$$
X^{\prime \prime}(t)+A(t) X(t)=0,
$$

where $A(t)=\left(a_{i j}(t)\right)$ is a symmetric $n \times n$ matrix and $X(t)=\left(X_{i}(t)\right)$ is an $n$-dimensional vector. Most of these results are established by using, either directly or indirectly, techniques from the calculus of variations. The assumption that $A$ is symmetric is essential since it is required in the application of the variational techniques. Recently, however, Ahmad and Lazer [2], [3], Ahmad [1], Schmitt and Smith [14], and Keener and Travis [10], using the theory of positive linear operators defined on a Banach space equipped with a cone, have obtained Sturmian type results for equation (1) which do not require the assumption that $A$ is symmetric (also see Gentry and Travis [7] and Keener and Travis [9] for related results). It is the purpose of this note to continue with these investigations and, in particular, to establish sufficient conditions for the oscillation of solutions to equation (1) which are valid when the matrix $A$ is nonsymmetric.

We will call the system (1) oscillatory on $[a, \infty)$ if for each $T>a$, there exist $\alpha, \beta>T$ and a nontrivial solution $X(t)$ of equation (1) satisfying $X(\alpha)=X(\beta)=0$. Recall that a number $b>a$ is said to be a conjugate point of $a$ if there exists a nontrivial solution $X(t)$ of equation (1) satisfying $X(a)=X(b)=0$. Equation (1) is said to be disconjugate on an interval $I$ if no nontrivial solution of (1) has more than one zero in $I$.

In order to relate our work to those mentioned in the bibliography, we will briefly discuss some other definitions of oscillation found in the literature. When $A$ is symmetric, our definition of oscillation is equivalent to the statement that for each $b \geqslant a, b$ has infinitely many conjugate points in the

Received by the editors October 10, 1977 and, in revised form, February 17, 1978.

AMS (MOS) subject classifications (1970). Primary 34C10.

Key words and phrases. Differential systems, oscillation. 
interval $[b, \infty)$. This equivalence can be verified as follows: assume that $b>a$, that equation (1) is oscillatory, and that there exist $b>a$ and $T_{0}>b$ such that $b$ has no conjugate points in the interval $\left[T_{0}, \infty\right)$. Let $\bar{X}(t)$ be the matrix solution of

$$
\bar{X}^{\prime \prime}(t)+A(t) \bar{X}(t)=0,
$$

satisfying $\bar{X}(b)=0$ and $\bar{X}^{\prime}(b)=I$, where $I$ is the identity matrix. If $\bar{X}\left(t_{0}\right)$ were singular for $t_{0}>T_{0}$, there would exist a nonzero vector $c$ such that $\bar{X}\left(t_{0}\right) c=0$, and hence the solution $y(t)=\bar{X}(t) c$ of equation (1) would vanish at $b$ and $t_{0}$, in contradiction to the assumption that $b$ has no conjugate points in the interval $\left[T_{0}, \infty\right)$. Thus $\bar{X}(t)$ is nonsingular on the interval [ $\left.T_{0}, \infty\right)$. If $W(t)=\bar{X}^{\prime}(t) \bar{X}^{-1}(t)$, it is easily verified that $W$ is a symmetric solution of the Riccati equation

$$
W^{\prime}(t)=-A(t)-W^{2}(t) .
$$

Therefore, equation (1) is disconjugate on $\left[T_{0}, \infty\right)$ (see [5] and [13]), and hence not oscillatory. Conversely, if we assume that for each $b>a, b$ has infinitely many conjugate points in the interval $[b, \infty)$, then clearly equation (1) is oscillatory.

Another definition of oscillation which has frequently appeared in the literature is that the determinant of every prepared matrix solution of equation (2) has arbitrarily large zeros. A matrix solution $\bar{X}$ of equation (2) is said to be prepared if

$$
\bar{X}^{*}(t) \bar{X}^{-1}(t)=\left(\bar{X}^{-1}(t)\right)^{*} \bar{X}(t)
$$

for all $t$ in $(a, \infty)$. When $A(t)$ is symmetric, this definition of oscillation is equivalent to the one we have given. To verify this, assume that the determinant of every prepared solution of equation (2) has arbitrarily large zeros. Then given $T>a$, there exists $\beta>\alpha \geqslant T$ such that the prepared solution of equation (2) satisfying $\bar{X}(\alpha)=0$ and $\bar{X}^{\prime}(\alpha)=I$ has a determinant of zero at $\beta$. Thus there exists a nonzero vector $c$ such that $\bar{X}(\beta) c=0$ and the solution $y(t)=\bar{X}(t) c$ of equation (1) vanishes at $\alpha$ and $\beta$. To prove the converse, let $\bar{X}(t)$ be a prepared solution of equation (2) and $y(t)$ a solution of equation (1) satisfying $y(\alpha)=y(\beta)=0$, where $\alpha$ and $\beta$ are arbitrarily large. Then the determinant of $\bar{X}$ is zero for some point in the closed interval $[\alpha, \beta]($ see $[11])$.

We recall that when $A(t)$ is a scaler function, a sufficient condition for the oscillation of equation (1) due to Wintner [19] is that

$$
\int_{a}^{\infty} A(s) d s=\infty
$$

Various generalizations of this result to selfadjoint systems have been obtained by Tomastik [17], [18], Kreith [11], Swanson [16], Noussair and Swanson [12], Kartsatos [8], Allegretto and Erbe [4], and Etgen and Pawlowski [6]. We give similar generalizations of Wintner's result to nonselfadjoint systems of the form (1). 
THEOREM 1. Assume that $A(t)=\left(a_{i j}(t)\right)$ is continuous on $(a, \infty)$ with $a_{i j}(t) \geqslant$ $0,1 \leqslant i, j \leqslant n$. If each column of $A(t)$ contains an element $a_{i j}(t)$ such that

$$
\int_{a}^{\infty} a_{i j}(s) d s=\infty
$$

then (1) is oscillatory.

We use the following theorem in our proof. Recall that an $n \times n$ matrix $A=\left(a_{i j}\right), 1<i, j \leqslant n$, is called irreducible if it is impossible to have $\{1,2, \ldots, n\}=I \cup J, I \cap J=\varnothing, I \neq \varnothing=J$, and $a_{i j}=0$ for all $i \in I$, $j \in J$.

Theorem 2. (See [1].) Let $A(t)=\left(a_{i j}(t)\right)$ be continuous on $(a, \infty)$ with $a_{i j}(t) \geqslant 0,1 \leqslant i, j \leqslant n$. If (1) is disconjugate on $(a, \infty)$, then there exists a nontrivial solution $\mu=\operatorname{col}\left(\mu_{1}, \ldots, \mu_{n}\right)$ of (1) such that $\mu(a)=0$ and $\mu_{i}(t) \geqslant 0$ for $i=1, \ldots, n$ and $t \geqslant a$. If, in addition, $A\left(t_{0}\right)$ is irreducible for some $t_{0}>a$, then $\mu_{i}(t)>0$ for $i=1, \ldots, n$ and $t>a$.

Proof of Theorem 1. Assume that (1) is not oscillatory on $(a, \infty)$. Then for some $T, T>a,(1)$ is disconjugate on $(T, \infty)$. By Theorem 2 , there exists a nontrivial solution $\mu=\operatorname{col}\left(\mu_{1}, \ldots, \mu_{n}\right)$ of (1) such that $\mu(t) \geqslant 0$ on $(T, \infty)$. Since for each $i, 1 \leqslant i \leqslant n$,

$$
\mu_{i}^{\prime \prime}(t)+\sum_{j=1}^{n} a_{i j}(t) \mu_{j}(t)=0,
$$

we have $\mu_{i}^{\prime \prime}(t) \leqslant 0$ for $t \geqslant T$. It follows that $\mu_{i}^{\prime}(t) \geqslant 0$ for $t \geqslant T$. For, if $\mu_{i}^{\prime}\left(t_{0}\right)<0$ for some $t_{0}, t_{0}>T$, then $\mu_{i}^{\prime}(t) \leqslant \mu_{i}^{\prime}\left(t_{0}\right)<0$ for $t \geqslant t_{0}$. But this would imply that $\mu_{i}(t) \rightarrow-\infty$ as $t \rightarrow \infty$, in contradiction to the fact that $\mu_{i}(t) \geqslant 0$. Now, since $\mu_{i}(t) \geqslant 0$ and $\mu_{i}^{\prime}(t) \geqslant 0$ on $(T, \infty)$, it follows that for each $i, 1 \leqslant i \leqslant n$, either $\mu_{i}(t) \equiv 0$ or $\mu_{i}(t)>0$ for $t>T$. Let $T_{0}>T$. Since $\mu(t)$ is a nontrivial solution, there exists an integer $j, 1 \leqslant j \leqslant n$, such that $\mu_{j}(t)>0$ for $t \geqslant T_{0}$. By hypothesis, there exists an integer $i, 1 \leqslant i \leqslant n$, such that

$$
\int_{T_{0}}^{\infty} a_{i j}(s) d s=\infty
$$

As $\mu_{j}(t)>0$ and $\mu_{j}^{\prime}(t) \geqslant 0$ on $\left(T_{0}, \infty\right)$, we can find a real number $k$ such that $0<k \leqslant \mu_{j}(t)$ for $t \geqslant T_{0}$. If we let $e_{j}$ be the $j$ th unit basis vector, then $k e_{j} \leqslant \mu(t)$ for $t \geqslant T_{0}$. Therefore,

$$
k A(t) e_{j}+\mu^{\prime \prime}(t) \leqslant A(t) \mu(t)+\mu^{\prime \prime}(t)=0,
$$

from which it follows that $k a_{i j}(t) \leqslant-\mu_{i}^{\prime \prime}(t)$, and consequently that

$$
k \int_{T_{0}}^{t} a_{i j}(s) d s-\mu_{i}^{\prime}\left(T_{0}\right) \leqslant-\mu_{i}^{\prime}(t)
$$

But since

$$
\int_{T_{0}}^{\infty} a_{i j}(s) d s=\infty
$$


it follows that $\mu_{i}^{\prime}(t) \rightarrow-\infty$ as $t \rightarrow \infty$, in contradiction to the fact that $\mu_{i}^{\prime}(t) \geqslant 0$ for $t \geqslant T_{0}$. This contradiction establishes the validity of the theorem.

CoROllary 1. If $a_{i j}(t) \geqslant 0,1 \leqslant i, j \leqslant n$, and

$$
\int_{a}^{\infty} a_{i i}(s) d s=\infty
$$

for each $i, 1 \leqslant i \leqslant n$, then equation (1) is oscillatory.

ReMARKs. If $A(t)=\left(a_{i j}(t)\right)$ is an $n \times n$ symmetric matrix with $a_{i j}(t) \geqslant 0$, $1 \leqslant i, j \leqslant n$, then a sufficient condition for the oscillation of equation (1) is that

$$
\int_{a}^{\infty} a_{i j}(s) d s=\infty
$$

for some pair $(i, j), 1 \leqslant i, j \leqslant n$ (see [4]). On the basis of this result, one might conjecture that Theorem 1 could be improved. To see that this is not the case without an additional hypothesis, consider equation (1) on the interval $(1, \infty)$ with $A$ defined by

$$
A(t)=\left(\begin{array}{cc}
\frac{1}{4 t^{2}}, & 0 \\
1, & \frac{1}{4 t^{2}}
\end{array}\right) .
$$

Let $K_{1}$ and $K_{2}$ be arbitrary constants, and let $g(t)$ be the general solution to

$$
Y^{\prime \prime}(t)+\frac{1}{4 t^{2}} Y(t)=-\sqrt{t}\left(K_{1}+K_{2} \ln t\right) .
$$

Then the general solution of equation (1) with $A$ given by (3) is

$$
\bar{X}(t)=\left(\sqrt{t}\left(K_{1}+K_{2} \ln t\right), g(t)\right) .
$$

If $K_{1}$ or $K_{2}$ is nonzero, then the first component has at most one zero. If $K_{1}=K_{2}=0$, then $g(t)=\sqrt{t}\left(\alpha_{1}+\alpha_{2} \ln t\right)$, and the second component has at most one zero. Thus, even though $\int_{1}^{\infty} a_{21}(s) d s=\infty$, equation (1) is nonoscillatory.

THEOREM 3. Assume that $A(t)=\left(a_{i j}(t)\right)$ is continuous on $(a, \infty)$ with $a_{i j}(t) \geqslant$ $0,1 \leqslant i, j \leqslant n$. Assume, further, that for each number $T, T \geqslant a$, there exists a number $t_{0}, t_{0}>T$, such that $A\left(t_{0}\right)$ is irreducible. Let $\Sigma A(t)$ denote the sum of all of the entries of the matrix $A(t)$. Then equation (1) is oscillatory if

$$
\int_{a}^{\infty} \sum A(s) d s=\infty
$$

Proof. Assume that (1) is not oscillatory on $(a, \infty)$. Then for some $T>a$, equation (1) is disconjugate on $(T, \infty)$. By Theorem 2 , there exists a nontrivial solution $\mu=\operatorname{col}\left(\mu_{1}, \ldots, \mu_{n}\right)$ of (1) such that $\mu(t)>0$ on $(T, \infty)$. In particular, $\mu_{j}(t)>0$ on $\left(T_{0}, \infty\right)$, where $T_{0}$ is any number such that 
$T_{0}>T$. Applying the same argument as in the proof of Theorem 1, we may conclude that $\mu^{\prime}(t) \geqslant 0$ for $t \geqslant T_{0}$. If we now let $e=\operatorname{col}(1,1, \ldots, 1)$, then we can find a real number $k$ such that $0<k e \leqslant \mu(t)$ for $t \geqslant T$. Therefore

$$
\begin{aligned}
k \sum A(t)+\sum_{i=1}^{n} \mu_{i}^{\prime \prime}(t) & =k(e, A(t) e)+\left(e, \mu^{\prime \prime}(t)\right) \\
& \leqslant\left(e, A(t) \mu(t)+\mu^{\prime \prime}(t)\right)=0 .
\end{aligned}
$$

The proof now follows from an argument similar to that used in the proof of Theorem 1.

COROllaRy 4. Assume $a_{i j}(t) \geqslant 0,1 \leqslant i, j \leqslant n$, and for each number $T \geqslant a$, there exists a number $t_{0}>T$ such that $A\left(t_{0}\right)$ is irreducible. Then equation (1) is oscillatory if

$$
\int_{a}^{\infty} a_{i j}(s) d s=\infty
$$

for some pair $(i, j), 1 \leqslant i, j \leqslant n$.

\section{REFERENCES}

1. S. Ahmad, On positivity of solutions and conjugate points of nonselfadjoint systems (to appear) (see Notices Amer. Math. Soc. 23 (1976), A-487, Abstract 76T-B134).

2. S. Ahmad and A. C. Lazer, On the components of extremal solutions of second order systems, SIAM J. Math. Anal. 8 (1977), 16-23.

3. __ An N-dimensional extension of the Sturm separation and comparison theory to a class of nonself-adjoint systems, SIAM J. Math. Anal. (to appear).

4. W. Allegretto and L. Erbe, Oscillation criteria for matrix differential inequalities, Canad. Math. Bull. 16 (1973), 5-10.

5. W. A. Coppel, Disconjugacy, Lecture Notes in Math., vol. 220, Springer-Verlag, Berlin and New York, 1971.

6. G. J. Etgen and J. F. Pawlowski, Oscillation criteria for second order self-adjoint differential systems, Pacific J. Math. 66 (1976), 99-110.

7. R. D. Gentry and C. C. Travis, Comparison of eigenvalues associated with linear differential equations of arbitrary order, Trans. Amer. Math. Soc. 223 (1976), 167-179.

8. A. G. Kartsatos, Oscillation of nonlinear systems of matrix differential equations, Proc. Amer. Math. Soc. 30 (1971), 97-101.

9. M. S. Keener and C. C. Travis, Focal points and positive cones for a class of nth order differential equations, Trans. Amer. Math. Soc. 237 (1978), 331-351.

10. __ Sturmian theory for a class of nonselfadjoint differential systems (to appear).

11. K. Kreith, Oscillation criteria for nonlinear matrix differential equations, Proc. Amer. Math. Soc. 26 (1970), 270-272.

12. E. S. Noussair and C. A. Swanson, Oscillation criteria for differential systems, J. Math. Anal. Appl. 36 (1971), 575-580.

13. W. T. Reid, Ordinary differential equations, Wiley, New York, 1971.

14. K. Schmitt and H. L. Smith, Positive solutions and conjugate points for systems of differential equations (to appear).

15. W. Simons, Disconjugacy criteria for systems of self-adjoint differential equations, J. London Math. Soc. 6 (1973), 373-381.

16. C. A. Swanson, Oscillation criteria for nonlinear matrix differential inequalities, Proc. Amer. Math. Soc. 24 (1970), 824-827.

17. E. C. Tomastik, Oscillation of nonlinear matrix differential equations of second order, Proc. Amer. Math. Soc. 19 (1968), 1427-1431.

18. Oscillation of systems of second order differential equations, J. Differential Equations 9 (1971), 436-442. 
19. A. Wintner, A criterion of ascillatory stability, Quart. Appl. Math. 7 (1949), 115-117.

Departuient of Mathimatics, Orelahoma State University, StmLlwater, Orchamoma 74074

Technology Assessuments Sbction, Health and Safety Research Division, Oax Ridge National laboratory*, P. O. BoX X, OAx Ridge, Tennesseb 37830

-Operated by the Union Carbide Corporation for the Energy Research and Development Administration. 